\title{
Children Only 3 Years Can Succeed At Conditional "If, Then" Reasoning, Much Earlier Than Anyone Had Thought Possible
}

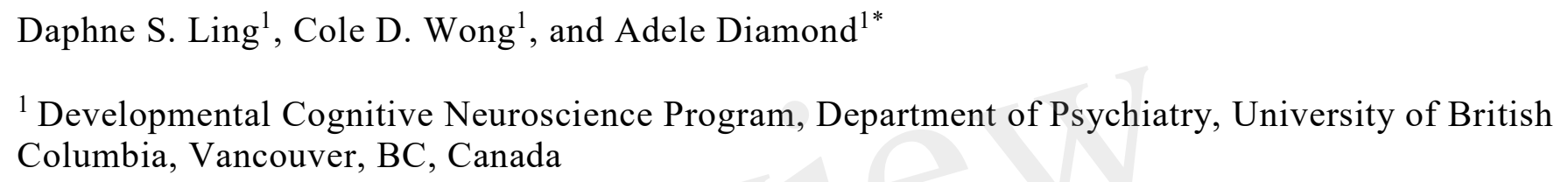

${ }^{1}$ Developmental Cognitive Neuroscience Program, Department of Psychiatry, University of British Columbia, Vancouver, BC, Canada

*Correspondence:

Prof. Adele Diamond

Developmental Cognitive Neuroscience Program

Department of Psychiatry

UBC

2255 Wesbrook Mall

Vancouver, BC Canada V6T 2A1

email: adele.diamond@ubc.ca

home phone during COVID: 6042662341

office phone: 6048227220
Number of words: 4547

Number of figures: 5

Number of tables: 1

Keywords: gestalt perception, perceptual pull2, preschoolers3, young children, conceptual understanding, conditional associative learning, dimensional change card sort (DCCS)7 


\section{Contribution to the Field Statement}

44 That conditional, if-then reasoning does not emerge until 4 to $4 \frac{1}{2}$ years of age has long been accepted.

45 Here we show that children barely 3 years old can do conditional reasoning. All that was needed was a

46 superficial change to the stimuli: When color was a property of the shapes (line drawings of a star and

47 truck) rather than of the background, 3-year-olds could deduce that red meant the star was correct and

48 blue that the truck was, thus succeeding at conditional discrimination. Three-year-olds do not seem to

49 use color to inform them which shape is correct unless color is a property of the shapes themselves,

50 suggesting they have difficulty mentally integrating dimensions that are not part of the same object. Our

51 findings suggest scaffolding preschoolers' emerging conceptual skills by changing the way stimuli look

52 (perceptual bootstrapping) enables 3-year-olds to demonstrate reasoning abilities long thought beyond

53 their grasp. These findings together with our earlier card-sort findings provide strong evidence against

54 prominent cognitive complexity, conditional reasoning, and graded memory theories for why 3-year-

55 olds fail conditional discrimination and card sorting. Perhaps the way we have traditionally queried

56 children has obscured the budding reasoning competencies present at 3 years of age. 


\begin{abstract}
We report results showing success at 3 years on conditional discrimination (CD) -- 12-18 months younger than previously reported. Three-year-olds succeeded when color was a property of the stimulus, rather than a property of the background, as in all past CD testing. Previously, we and others found children succeed on the dimensional change card sort (DCCS) test at 3 years -- 12-24 months earlier than previously reported -- by making color a property of the background, instead of a property of the stimulus, as in standard DCCS testing. Neither the change to CD or DCCS affected the rule structure or reasoning requirements of the task. This double dissociation, with 3-year-olds performing better on $\mathrm{CD}$ when color and shape were integrated but better on DCCS when color and shape were separated, indicates that when superficial stimulus properties are modified 3-year-olds can do conditional reasoning and grasp a hierarchical rule structure - but they seem to need perceptual boot-strapping to do that. Children of 3 years evidently have difficulty mentally separating physical dimensions (e.g., color and shape) of the same object and difficulty mentally integrating physical dimensions not part of the same object. These results provide the strongest evidence to date against conceptual accounts of why children of 3 years fail conditional discrimination or card sorting.
\end{abstract}


In conditional discrimination (CD) tasks with children (Gollin and Liss, 1962; Gollin, 1965; Andrews et al., 2012), which response is correct is conditional on which of two colors is present: Shape A is correct when Color 1 is present and Shape B is correct when Color 2 is present. Psychologists have assumed for over 50 years that the ability to do conditional, if-then reasoning does not develop until roughly 4 to 5 years of age, since children younger than that have consistently failed $\mathrm{CD}$. We show here, however, that children only 3 years old can succeed at CD (that is, are capable of conditional, if-then reasoning) when superficial stimulus properties are modified. To succeed at a CD task, a child must integrate the two dimensions (color and shape), yet psychologists have traditionally presented CD tasks as if the two dimensions were completely separate. Instead of making color a property of the background or of the outside border on stimulus cards (as in all previous CD experiments with children), we made color a property of the stimuli themselves (the shapes were either Color 1 or Color 2). This manipulation allowed children of 3 years to succeed.

Card sorting (as in the Dimension Change Card Sort [DCCS] task), on the other hand, requires attending only to color information when that dimension is relevant (ignoring shape) or attending only to shape information when that dimension is relevant (ignoring color) and being able to switch from doing one to the other. Correct sorting thus requires a child to separate the same two dimensions that $\mathrm{CD}$ requires a child to integrate. Depending on which dimension (shape or color) is currently relevant for sorting the cards, children are supposed to ignore the other. Until we (Diamond et al., 2005) and Kloo and Perner (2005) came along, psychologists had always presented card sorting tasks to children with both color and shape as properties of each stimulus object (e.g., a blue star or a red truck drawn on a stimulus car). Based on the repeated failure of children younger than $4 \frac{1}{2}$ to 5 years on the DCCS task, many had concluded that children younger than $4 \frac{1}{2}$ years are not capable of conditional reasoning or grasping a hierarchical rule structure (e.g., Andrews et al., 2012; Zelazo et al., 2003). We hypothesized that if color was a property of the background instead of a property of the stimulus as in standard DCCS testing that children would be able to successfully switch sorting dimensions at a younger age, and indeed that is what we found (2003). That simple manipulation enabled children to succeed on the DCCS test at 3 years -- 12-18 months earlier than previously reported. Thus separating color and shape in the visual display aided 3-year-olds in conceptually ignoring one dimension when the task required that they focus on the other.

Our hypothesis here is that integrating color and shape in the visual display when the task requires conceptually integrating those dimensions (as does CD) should enable 3-year-olds to succeed because it bootstraps the children perceptually in their task of conceptually relating the two dimensions to one another. We tested that and present here the first demonstration that the age of first success on CD can be reduced from 4-5 years to 3 years by a surface modification of the stimuli. When color is a property of the stimulus object (i.e., color and shape are integrated as properties the same object), instead of color appearing as part of the background, 3-year-olds can succeed at CD.

\section{Participants}

\section{METHODS}

A total of 42 children were tested. All children could understand and converse in English, had normal or normal-with-correction hearing and sight. None were taking any medication that affected cognition. None had suffered a concussion or lost consciousness from a fall or blunt trauma to the head. This study was approved by the UBC Behavioural Research Ethics Board (REB\# H04-80913) and Vancouver Coastal Health (V12-80913). A parent or guardian of each child gave written informed consent for the child's participation. 
Participants were recruited from two age groups: children almost or just barely 3 years old and children almost or just barely 4 years old. They were tested in a StrongStart Centre in the greater Vancouver area $(\mathrm{N}=25)$ or in our lab at UBC $(\mathrm{N}=17)$. All children were accompanied by a parent, grandparent, or caregiver. The adult chaperone sat behind the child during testing or watched through the lab's one-way mirror. A random subset of sessions was videotaped with permission from the parent or caregiver.

Six children ( 5 girls and 1 boy) were excluded from data analyses because they appeared to be unable to grasp how the task worked (that they were to retrieve rewards) or were not interested in it. Five of these children were 3 years old ( 3 tested on integrated, 2 on separated [including the one boy]). The sixth child was a 4-year-old girl tested on separated dimensions.

Our data set thus consists of 36 children, 22\% were Caucasian, 22\% East Asian, 8\% Hispanic, $6 \%$ South Asian, 11\% Mixed Ethnicity, and 6\% were other, and 25\% did not report their ethnicity. Most children $(75 \%)$ came from a home where the primary caregiver has a college degree. In the 3-year-old age group, there were 24 children ( $44 \%$ female). Half were tested on CD with integrated dimensions and half with separated dimensions. The mean age for the 3 -year-olds was 3.1 years $(\mathrm{SD}=0.16$ years; range $=33.5$ - 39.5 months). See Table 1.

In the 4-year-old age group, there were 12 children ( $33 \%$ female). They were tested on separated dimensions to see if, when we used the same procedure as have previous studies from other labs, we would get the same results. The mean age for the 4 -year-olds was 4.0 years $(\mathrm{SD}=0.22$ years; range $=45.5-53.0$ months). See Table 1. A priori power analyses indicated that for a medium effect size of 0.35 ( $80 \%$ power), 12 children per group was sufficient.

Insert Table 1 about here

\section{Materials}

A child sat directly across from the experimenter at a table measuring $76 \times 76 \times 55 \mathrm{~cm}$. The child was seated in a child-sized chair $(36 \times 30 \times 36 \mathrm{~cm})$ and the experimenter was seated on a stool $(20 \times 39 \times$ $23 \mathrm{~cm}$ ). Two rectangular wooden boxes open at the top, each measuring $12.5 \times 8.6 \times 3.7 \mathrm{~cm}$, served as the containers where the reward was hidden. These boxes were identical in appearance. At the base of one of the boxes on the inside was a marble well. The marble well held the marble in place to prevent the child from guessing the marble's location based on the sound of the marble rolling around. The stimulus cards served as the boxes' lids. The cards for both conditions measured $13.3 \times 9.6 \mathrm{~cm}$ and were laminated. For the integrated condition, the stimulus cards displayed a star or a truck that was either blue or red on a white background. For the separated condition, the shapes (star or truck) were white outlined in black and the border of the cards was either blue or red. See Figure 1.

\section{Insert Figure 1 about here}

Marbles ( $1.5 \mathrm{~cm}$ diameter) of different colors and patterns served as the reward. When children found a marble, they could put it in our marble maze and watch as the marble soared down ramps and spun through turnstiles. The marble maze (see Figure 2) stood $28.0 \mathrm{~cm}$ tall and was on a flat platform measuring $20.0 \times 15.5 \mathrm{~cm}$. A plastic, transparent jar $(6.5 \times 6.5 \times 11 \mathrm{~cm})$ was used to display the trove of marbles a child had found. 
170

171

172

173

174

175

176

177

178

179

180

181

182

183

184

185

186

187

188

189

190

191

192

193

194

195

196

197

198

199

200

201

202

203

204

205

206

207

208

209

210

211

212

213

214

215

216

217

\section{Testing Procedure}

First, the experimenter showed the child where to sit. The parent/guardian was given the option of sitting directly behind the child or watching from outside the testing room through a one-way mirror.

At the outset of testing, the experimenter told the child they were going to play a game and asked the child to cover his or her eyes like in the game "Peekaboo" ("I am going to bring out a surprise. Can you cover your eyes, like this?" [the experimenter showed child]). While the child's eyes were closed and covered, the experimenter placed a marble in the marble well inside one of two boxes; this was the done underneath the table, out of sight even to the parent. Then the experimenter covered each box with a stimulus card (one showing a truck, the other showing a star) and placed the two boxes on the table, one to the left and one to the right, both equidistant from the child, and within the child's reach. The left-right locations of the correct stimulus card was varied in the same pseudo-random sequence for all sessions (see below). The child sat, eyes covered, waiting in anticipation. The experimenter then announced: "You can open your eyes now. I have hidden a surprise for you under one of these cards. Can you guess which one?"

The child was encouraged to choose a card and lift it to see if the surprise was hidden beneath. If a child chose the correct card, the experimenter cheered exuberantly and encouraged the child to retrieve the hidden marble. The child was then presented with the marble maze and shown how to place the marble in it, to the enormous delight of the child.

If the child chose the wrong card, the experimenter said disappointedly, "Oh no, it wasn't there." The experimenter then lifted the correct card and showed the child where the reward had been hidden and said, "It was here, see? Let's try again. You'll find it next time!" The child was thus given feedback on each trial and the experimenter either cheered happily or showed the child what the correct choice had been and encouraged the child to find the marble next time. At no point did the experimenter ever explicitly state that the marble was under the truck or star or state the conditional rule. In Block 1, all the cards contained blue. The marble was always hidden under the truck card. The right-left location of the stimulus cards was pseudo-randomly varied across trials (Truck: Left, L, Right, L, R, R, L, R, L, L, R, R - repeated as long as needed up to a total of 36 trials). Participants never saw the reward being hidden and were never explicitly told the rules of the game. To find the reward they had to deduce the rule governing where it would be. As the right $(\mathrm{R})$ and left $(\mathrm{L})$ locations of the stimuli were randomly varied, always reaching right or left as a strategy did not lead to success. Six consecutively correct trials were required to pass the block and move on to the next one.

In Block 2, all cards contained red. Here, the marble reward was always hidden under the star stimulus card. Again, the right-left location of the stimulus cards was pseudo-randomly varied (Star: L, R, $R, L, R, L, L, R, L, R, R, L$ - repeated as long as needed up to a total of 36 trials). The child needed to pick correctly on six trials in a row to pass criterion and move on to the final block.

On the first and second block, a child did not need to pay attention to color to choose correctly. The truck was always the correct choice for Block 1 and the star was always the correct choice for Block 2. There was no need to integrate color and shape information; attending to shape alone was sufficient.

In Block 3, cards containing blue or red were randomly intermixed over trials. On any given trial, both cards contained red or both contained blue. Again, the truck was the correct choice when both cards contained blue and the star was correct when both cards contained red. The following pseudo-random 
order indicates which color was presented on which trial and whether the correct choice was presented on the right or left: Blue + Left, Red + Right, Red + Left, Blue + Left, Red + Right, Blue + Right, Blue + Right, Red + Left, Blue + Right, Red + Right, Blue + Left, Red + Left. This was repeated as long as needed up to a maximum of 36 trials. As with Blocks 1 and 2, the criterion for passing Block 3 was six correct trials in a row.

For all hypotheses, the block of interest was Block 3. That is the only block where conditional reasoning was required - if red is present, choose the star, if blue is present, choose the truck. Our hypotheses were: (1) First and foremost, children of 3 years would succeed on CD (i.e., succeed on Block 3 ) with the dimensions of color and shape integrated in the stimuli. Since we predicted that children of 3 years would perform roughly as well on CD (i.e., roughly as well on Block 3) with integrated dimensions as children of 4 years perform on $C D$ with separated dimensions, we defined success on $C D$ as roughly comparable Block 3 performance by 3-year-olds on integrated dimensions as 4-year-olds show on separated dimensions. (2) Children of 3 years would perform significantly better on CD (i.e., on Block 3 ) when color and shape were integrated in the stimuli than when they were separated. (3) We would replicate previous findings that (a) children of 3 years would fail CD (i.e., fail to reach criterion in Block 3 ) when color and shape are separated on the stimulus cards, (b) children of 4 years would succeed on CD (i.e., succeed on Block 3) with that same condition (separated dimensions), i.e., they would perform roughly comparably on our CD task with separated dimensions as other labs have found 4-year-olds to perform on CD with separated dimensions when they tested that, and (c) in all conditions and at both ages children would succeed on Blocks 1 and 2.

The criterion for passing a block was 6 correct responses in a row, for which we gave each child up to 18 trials per block. The choice of 18 trials was based on the work of Gollin and Liss (1962), who used 16-20 as their cut-off for Block 3 in their CD testing, after which the experimenter stepped in to aid the child in picking the correct stimulus. For each of age $\mathrm{X}$ task group, we analysed the number of trials needed to succeed on 6 trials in a row as well as the percentage of children who did so in 18 trials or less. We let children continue to try to figure out the CD rule after 18 trials, but only considered a child as having succeeded on a block if 6 correct trials in a row occurred within 18 trials or less. Our third dependent measure, however, was the average number of trials performed by each age $\mathrm{X}$ task group.

\section{RESULTS}

Since neither tester, gender, nor tester $\mathrm{X}$ gender was significantly related to any dependent variable for any test of our hypotheses, we dropped those variables from our statistical tests. Analysis of variance (ANOVA) was used to test all hypotheses except for hypotheses concerning the binary variable of pass/fail, for which Fisher's exact test was used.

Hypothesis 1: We turn first to our principal hypothesis, that children only 3 years old would succeed on CD with integrated dimensions. We operationalized "success" as performance of 3-year-olds on CD with integrated dimensions that is comparable to 4-year-olds on CD with separated dimensions. The percentage of 3-year-olds passing Block 3 of the integrated condition of CD (75\%) was identical to the percentage of 4-year-olds passing Block 3 of the canonical version of the task (75\%). See Figure 3. Children of 3 years took an average of 14.2 trials $(\mathrm{SD}=7.8)$ to pass Block 3 when color and shape were integrated in the stimuli. Children of 4 years took an average of 16.3 trials $(\mathrm{SD}=9.5)$ to succeed on the standard CD task when color and shape were separated in the stimuli. The number of trials needed to pass Block 3 was not significantly different between the two groups $(F(1,22)=0.36$, NS). Indeed, if anything, the number of trials was slightly lower for 3-year-olds with integrated dimensions than for 4-year-olds with separated dimensions. See Figure 4. We conclude that Hypothesis 1 was confirmed. 
Insert Figures 3 and 4 about here

Hypothesis 2: We predicted that 3-year-olds would performed better on the integrated-dimensions condition than on the separated-dimensions condition. The percentage of 3-year-olds passing the integrated condition of $\mathrm{CD}(75 \%)$ was greater than the percentage of 3-year-olds passing the separated condition of CD (33\%; Fisher's Exact Test, $p=.05)$. See Figure 4 above. Children of 3 years tested with separated dimensions (where color was a property of the border) took an average of 23.5 trials $(\mathrm{SD}=9.8)$ to pass Block 3, whereas those tested with integrated dimensions (where color was a property of the truck or star) took an average of only 14.2 trials $(\mathrm{SD}=7.8)$ to pass Block 3 . That difference is significant: $\mathrm{F}(1$, $22)=6.71, \mathrm{p}<0.02$ (effect size: 0.23). See Figure 3. We conclude that Hypothesis 2 was confirmed.

Hypothesis 3a: We had predicted that we would replicate findings that 3-year-olds tested on CD with separated dimensions would fail. Only $33 \%$ of the 3 -year-olds tested with separated dimensions passed Block 3. Thus, most children of 3 years tested with the canonical version of CD failed $(67 \%)$.

Hypothesis 3b: We predicted that when presented with the canonical CD task with separated dimensions, we would replicate previous findings that 4-year-olds would succeed. Our results show that $75 \%$ of 4 -year-olds tested o CD with separated dimensions succeeded. This is similar to what Gollin (1962) found, which was that $68 \%$ of 4 -year-olds succeeded. See Figure 4.

Hypothesis 3c: Lastly, we predicted that in all conditions and at both ages, children would succeed on Blocks 1 and 2 as these blocks are fairly easy. In both Blocks 1 and 2, color was irrelevant because on all trials both cards contained the same color (Block 1: Blue; Block 2: Red). Indeed, all children tested, regardless of age or condition, passed Blocks 1 and 2. Children of 3 years tested in the integrated condition took 10.2 and 13.3 trials respectively to pass Blocks 1 and 2 . Children of 3 years tested in the canonical separated-dimensions condition took 12.5 and 16.0 trials respectively to pass Blocks 1 and 2. Children of 4 years tested in the canonical separated-dimensions condition took an average of 10.3 and 11.2 trials respectively to pass Blocks 1 and 2. We conclude that all three sub-components of Hypothesis 3 were confirmed

Our results provide evidence that integrating the dimensions allows children to perform CD at a level 12 months ahead of when most had previously thought possible. Like others before us, we found that 3-year-olds fail and 4-year-olds succeed at CD presented the canonical way with color and shape separated on the stimulus cards (Andrews et al., 2012; Gollin, 1965; Gollin and Liss, 1962; Rudy et al., 1993).

\section{DISCUSSION}

We hypothesized that children of only 3 years are capable of conditional, if-then reasoning, but they do not seem to mentally integrate dimensions that are not properties of the same object. That is, when performing the CD task, they do not appreciate that the color in the background is telling them anything about which shape is the correct choice. On CD tasks children need to use the color shown to inform them which shape is correct (i.e., it is critical that they integrate color and shape information). We report here that when the dimensions of color and shape are integrated as part of the same stimulus, children of 3 years ( $\geq 12$ months younger than previously reported) can use the value on one dimension (color) to indicate which value of the other dimension (shape) is correct, and thus succeed at CD. When color and shape are separated on the stimulus cards, 3-year-olds do not seem to comprehend that color is telling them anything about which shape is correct. 
314

315

316

317

318

319

320

321

322

323

324

325

326

327

328

329

330

331

332

333

334

335

336

337

338

339

340

341

342

343

344

345

346

347

348

349

350

351

352

353

354

355

356

357

358

359

360

361
The current finding may be thought of as the flip-side of what we (Diamond et al., 2005) and Kloo and Perner (2005) found with the DCCS task. Children under 4 or 5 years typically fail to switch dimensions on the DCCS. We hypothesized that if color and shape were not part of the same object, but instead if colorless shapes (black or white) were presented on cards with a background color, that children only 3 years old would be able to switch from sorting by color to sorting by shape or vice versa. Our hypothesis was confirmed (Diamond et al., 2005) and soon thereafter Kloo and Perner showed the same thing with colorless shapes and a color patch on each card. Children of 3 years can switch sorting dimensions when the dimensions are perceptually separate and not part of the same object.

The present findings together with those just cited for DCCS present the strongest evidence to date against several of the most prominent theories proposed for why 3-year-olds fail CD or DCCS. Evidently, 3-year-olds can grasp the hierarchical rule structure of the task (unlike Zelazo's influential Cognitive Complexity and Control - Revised [CCC-R] hypothesis; Zelazo et al., 2003), have sufficient memory (unlike Munakata's influential graded memory hypothesis; Morton and Munakata, 2002), and are quite capable of conditional, if-then reasoning (unlike Halford's influential hypothesis; Andrews et al., 2012; Halford et al., 1998, Halford et al., 2010's proposition) since when superficial stimulus properties were changed, children of 3 years succeeded.

The perceptual bootstrap we provided through changing superficial properties of the stimulus cards removed neither the need to grasp the embedded hierarchical rule structure, the memory demands of the task, nor the need for conditional "if, then" reasoning. Our study therefore suggests that scaffolding preschoolers' emerging conceptual skills by changing the way the stimuli look (perceptual bootstrapping) enables 3-year-olds to demonstrate if-then conceptual reasoning abilities long thought beyond their grasp.

The results reported here for conditional discrimination combined with those of Diamond et al. (2005) and Kloo and Perner (2005) on DCCS present a clear double dissociation between how integrating or separating the dimensions of color and shape affect the performance of 3-year-olds. What helps performance on CD hinders performance on DCCS and what helps performance on DCCS hinders performance on CD. Figure 5 symbolically displays this dissociation.

\section{Insert Figure 5 about here}

While both the CD and DCCS tasks involve conditional reasoning, CD requires the integration of dimensions while DCCS requires the separation of those same two dimensions. Could it be that the way we have traditionally queried children has not made it possible for 3-year-olds to show evidence of their budding reasoning competencies?

We acknowledge that there are limitations to our study. Our sample sizes were relatively small - 12 per group. Our results are clear, however, and our number of subjects sufficient to find significant results. Sample sizes used here are quite comparable to those used by Kloo and Perner (2005) for testing separated versus integrated dimensions on the DCCS task. In one of their experiments, conditions were tested between-subjects (as here) and they had 12 participants per group (as here). In another of their experiments, conditions were tested within-subject with only nine participants per group. Another limitation is that we tested only one task (CD). It would have been more elegant to test the same children on both CD and DCCS. It would also have been more elegant to test each 3-year-old on both versions of $\mathrm{CD}$, but we learned in using that design with DCCS that there was spillover from the easier condition to the more difficult one when tested within-child (i.e., children did better on DCCS with integrated 
362

363

364

365

366

367

368

369

370

371

372

373

374

375

376

377

378

379

380

381

382

383

384

385

386

387

388

389

390

391

392

393

394

395

396

397

398

399

400

401 dimensions when that was tested second than when it was tested first; Diamond et al., 2005). Also, CD testing takes longer than DCCS; almost none of the children we tested would have sat through another session testing the other $\mathrm{CD}$ condition. Another limitation is that our testers were not blind to our hypotheses as they had helped design the study, so it is always possible that they might have subtly, unintentionally affected children's performance. Finally, we only tested children of 4 years on the integrated dimensions version of $\mathrm{CD}$. While this last point might look like a limitation, since others had shown that 4-year-olds succeed on the more difficult version of CD (separated dimensions) it seemed unnecessary to test them on the easier version of the task (integrated dimensions).

Our results are consistent with those of other studies that used other paradigms. Jarvik's (1956) study shows perhaps the most astonishing evidence. Many studies had shown that it takes chimpanzees over 100 trials to learn a simple visual discrimination (e.g., choose the red or green stimulus) when the reward is just below the stimulus card in a shallow well. Jarvik varied whether the reward was hidden 0.1 $\mathrm{cm}$ below the stimulus card in a shallow well or whether it was taped to a depression in the underside of the stimulus card. He replicated the result that with the reward in the well just below the stimulus card it takes chimpanzees an average of 131 trials to learn a visual discrimination. However, Jarvik found that chimpanzees were able to learn visual discriminations in only one trial when the reward was attached to underside of the stimulus.

Our lab has previously shown the importance of proximity-based perceptual modifications in other studies. When rewards were physically connected to the stimulus objects (e.g., by Velcro or even a string some inches long), infants only 9-12 months old could successfully use the stimuli to guide them to learn a delayed non-matching rule (choose the stimulus that does not match the sample you were just previously shown; (Diamond et al., 1999; Shutts et al., 2001). When the reward is not attached to the stimulus object, but in the well just below, as in the canonical delayed non-matching to sample task, toddlers cannot succeed until they are 18-21 months old (Diamond, 1990; Overman, 1990; Diamond et al., 1994).

Deloache's lab has likewise found results consistent with this: They report that toddlers of 18-22 months are significantly more likely to retrieve a reward they saw hidden when it is hidden inside a piece of furniture than if it is hidden near the same piece of furniture (DeLoache and Brown, 1983). Toddlers of 21 months can find a hidden object if it is hidden inside one of 4 attractive containers but they cannot use those same attractive containers to inform them where to search when those containers are mounted on top of four identical plain boxes (DeLoache, 1986).

In conclusion, if children of 3 years can succeed at $\mathrm{CD}$ when color and shape are integrated as part of the stimulus, then they must be capable of if-then, conditional reasoning at some level. The ability might be fragile, but it appears to be present. Children of 3 years seem to rely on perceptual information (physical characteristics of the stimuli) to guide them in appreciating that the value of one dimension (color) is informing them about which value of the other dimension (shape) is correct. 
Earlier Success at Conditional Discrimination

402

403

404

405

406

407

408

409

410

411

412

413

414

415

416

417

418

419

420

421

422

423

424

425

426

427

428

429

430

431

432

433

434

435

436

437

438

439

440

441

442

443

444

445

446

447

448

449

\section{REFERENCES}

Andrews, G., Halford, G.S., and Boyce, J. (2012). Conditional discrimination in young children: The roles of associative and relational processing. Journal of Experimental Child Psychology 112, 84-101. doi: 10.1016/j.jecp.2011.12.004.

DeLoache, J.S. (1986). Memory in very young children: Exploitation of cues to the location of a hidden object. Cognitive Development 1, 123-137. doi: 10.1016/S0885-2014(86)80015-6.

DeLoache, J.S., and Brown, A.L. (1983). Very young children's memory for the location of objects in a large-scale environment. Child Development 54, 888-897. doi: 10.1111/j.14678624.1983.tb00510.x.

Diamond, A. (1990). Rate of maturation of the hippocampus and the developmental progression of children's performance on the delayed non-matching to sample and visual paired comparison tasks. Annals of the New York Academy of Sciences 608, 394-426.

Diamond, A., Carlson, S.M., and Beck, D.M. (2005). Preschool children's performance in task switching on the dimensional change card sort task: Separating the dimensions aids the ability to switch. Developmental Neuropsychology 28, 689-729. doi: 10.1207/s15326942dn2802_7.

Diamond, A., Churchland, A., Cruess, L., and Kirkham, N.Z. (1999). Early developments in the ability to understand the relation between stimulus and reward. Developmental Psychology 35, 1507-1517.

Diamond, A., Towle, C., and Boyer, K. (1994). Young children's performance on a task sensitive to the memory functions of the medial temporal lobe in adults, the delayed nonmatching-to-sample task, reveals problems that are due to non-memory-related task demands. Behavioral Neuroscience 108, 659-680. doi: 10.1037/0735-7044.108.4.659.

Gollin, E.S. (1965). Factors affecting conditional discrimination in children. Journal of Comparative and Physiological Pyschology 60, 422-427. doi: 10.1037/h0022583.

Gollin, E.S., and Liss, P. (1962). Conditional discrimination in children. Journal of Comparative and Physiological Psychology 55, 850-855. doi: 10.1037/h0045951.

Halford, G.S., Wilson, W.H., and Phillips, S. (1998). Processing capacity defined by relational complexity: Implications for comparative, developmental, and cognitive psychology. Behavioral and Brain Sciences 21, 803-831, discussion; 831-864, review. doi: 10.1017/S0140525X98001769.

Halford, G.S., Wilson, W.H., and Phillips, S. (2010). Relational knowledge: The foundation of higher cognition. Trends in Cognitive Sciences 14, 497-505. doi: 10.1016/j.tics.2010.08.005.

Jarvik, M.E. (1956). Simple color discrimination in chimpanzees: Effect of varying contiguity between cue and incentive. Journal of Comparative and Physiological Psychology 49, 492-495. doi: $10.1037 / \mathrm{h} 0045644$.

Kloo, D., and Perner, J. (2005). Disentangling dimensions in the dimensional change card sorting task. Developmental Science 8, 44-56. doi: 10.1111/j.1467-7687.2005.00392.x.

Morton, J.B., and Munakata, Y. (2002). Active versus latent representations: A neural network model of perseveration and dissociation in early childhood. Developmental Psychobiology 40, 255-265. doi: 10.1162/0898929053467622.

Overman, W.H. (1990). Performance on traditional match-to-sample, nonmatch-to-sample, and object discrimination tasks by 12 to 32 month-old children: A developmental progression. Annals of the New York Academy of Sciences 608, 365-393.

Rudy, J.W., Keith, J.R., and Georgen, K. (1993). The effect of age on children's learning of problems that require a configural association solution. Developmental Psychobiology 26, 171-184.

Shutts, K., Ross, E., Hayden, M., and Diamond, A. (Year). "Grasping that one thing is related to another: Contributions of spatial contiguity, temporal proximity, and physical connection", in: Biennial Meeting of the Society for Research in Child Development). 
450 Zelazo, P.D., Müller, U., Frye, D., and Marcovitch, S. (2003). The development of executive function in 451 early childhood. Monographs of the Society for Research in Child Development 68(3), 1-137. 
Earlier Success at Conditional Discrimination

454

455

456

457

458

459

460

461

462

463

464

465

466

467

468

469

470

471

472

473

474 475

Table 1

Age, Number, and Genders of Children in Each Group

\begin{tabular}{ccccccc} 
Condition & Age & Mean Age & SD Age & Male & Female & $\mathrm{n}$ \\
\hline Integrated & $2.8-3.3$ years & 3.07 years & 0.16 & 6 & 6 & 12 \\
Separated & $2.8-3.3$ years & 3.05 years & 0.16 & 6 & 6 & 12 \\
Separated & $3.8-4.4$ years & 4.03 years & 0.22 & 9 & 3 & 12 \\
\hline
\end{tabular}

\section{Figure Captions}

Figure 1: Examples of standard (separated) and integrated conditional discrimination stimuli used in the task.

Figure 2: Marble maze used in the study.

Figure 3: Graph comparing performance of 3- and 4-year-olds in Gollin's Conditional Discrimination (1965) with the current study.

Figure 4: Graph comparing performance of 3- and 4-year-olds on Block 3 of the Conditional Discrimination Task in the current study.

Figure 5: Graph showing the double dissociation of performance in the dimensional change card sort and conditional discrimination in 3-year-olds. 


\section{Conflict of Interest}

477 The authors declare that the research was conducted in the absence of any commercial or financial 478 relationships that could be construed as a potential conflict of interest.

\section{Author Contributions}

DSL actively contributed to all aspects of this study and manuscript preparation.

CDW contributed to recruiting and testing the participants, entering the data, and thinking about what the results meant.

AD actively contributed to all aspects of this study and manuscript preparation, except testing participants.

\section{Funding}

DSL gratefully acknowledges financial support from the Canadian Institutes of Health Research Frederick Banting and Charles Best Canada Graduate Scholarship (CGS-D) to Honour Nelson Mandela award and the UBC Institute of Mental Health Marshall Scholarship.

AD gratefully acknowledges financial support from NIDA R01 \# DA019685 for planning and conducting the study. AD further acknowledges that support from Canada Research Chair award \#CRC950-27472 and a grant from the Bezos Family Foundation to AD helped fund preparation of this manuscript.

\section{Acknowledgments}

505 greater Vancouver area, and the children and their families who participated in this research. 
Figure 1
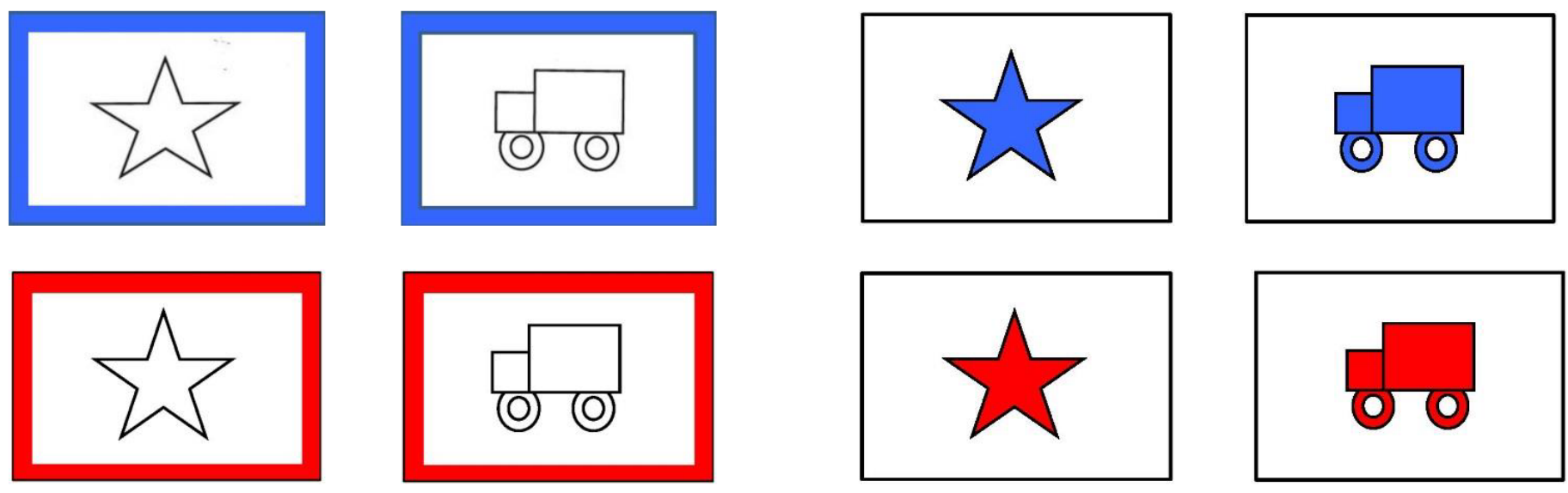

Example of standard (separated)
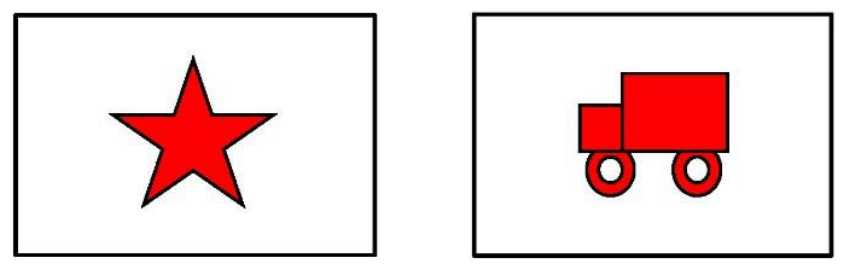

Example of integrated conditional

conditional discrimination stimuli

discrimination stimuli 
Figure 2.TIFF

\section{Figure 2}

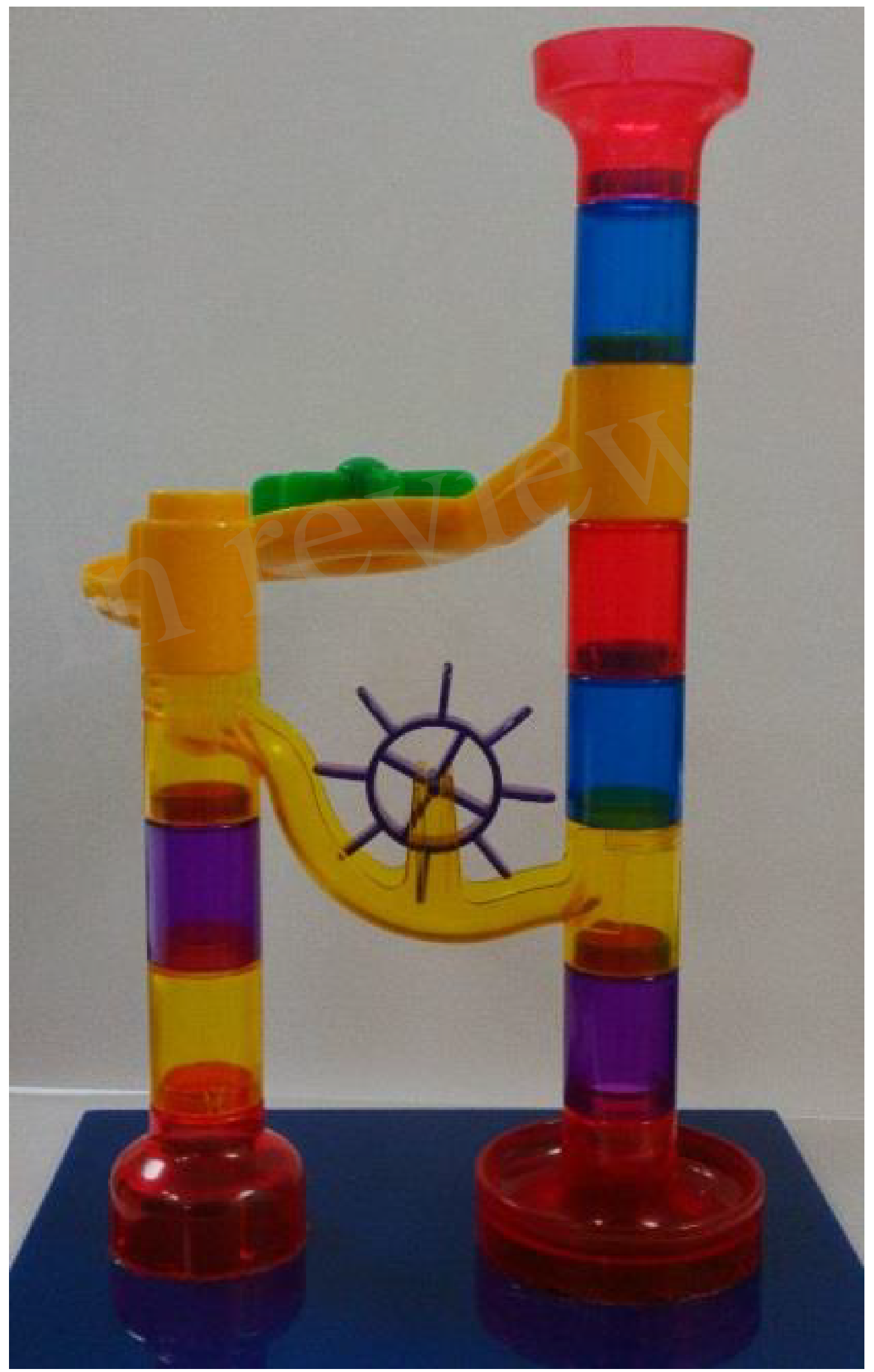




\section{Figure 3}

\section{Results Comparing Performance of 3- and 4-year-olds in Gollin's Conditional Discrimination (1965) with the current study}

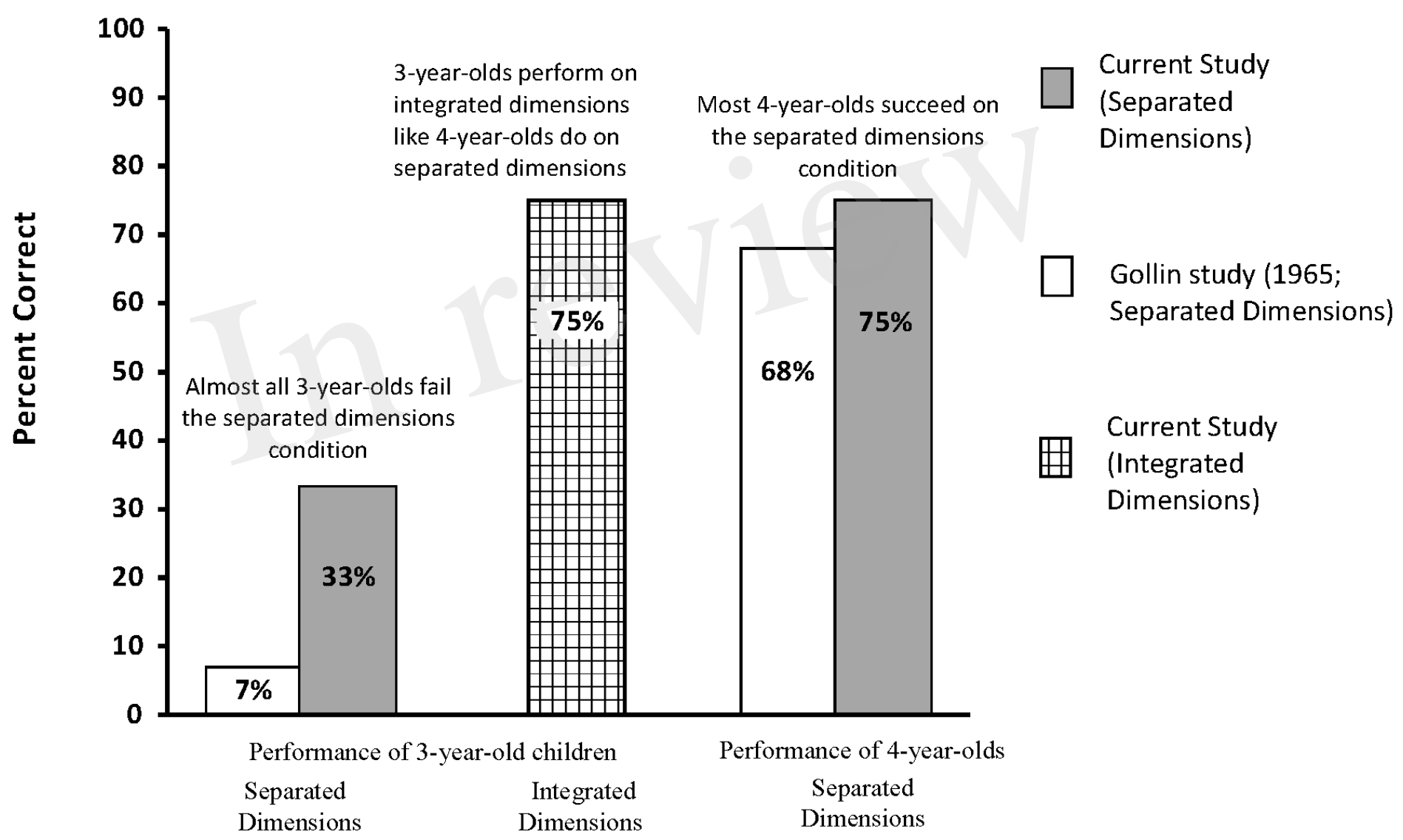


Figure 5

Performance of 3-year-olds on the Dimensional Change Card Sort (Diamond et al., 2005) and Conditional Discrimination (current study)

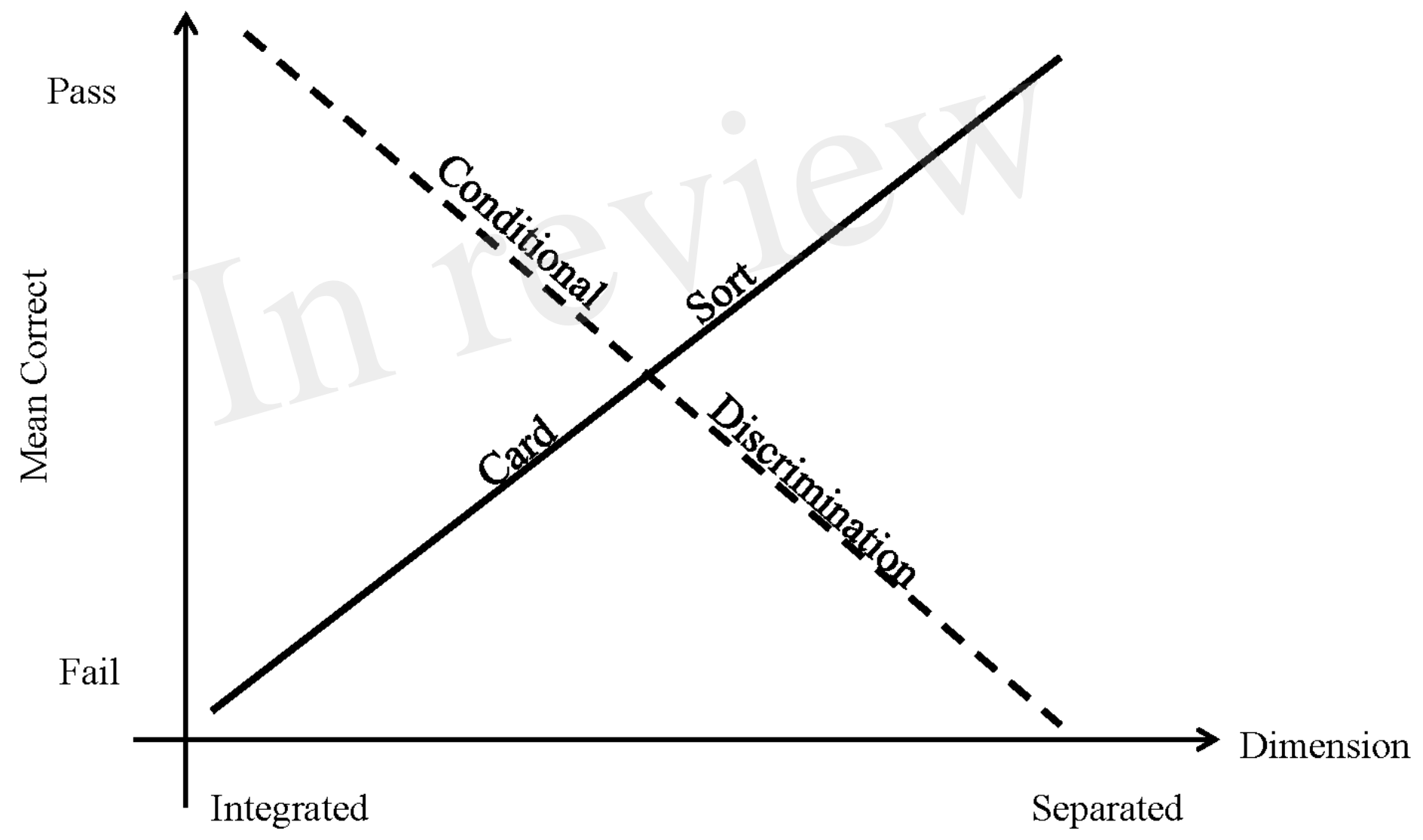


Figure 4

Performance on Block 3 of the Conditional Discrimination Task

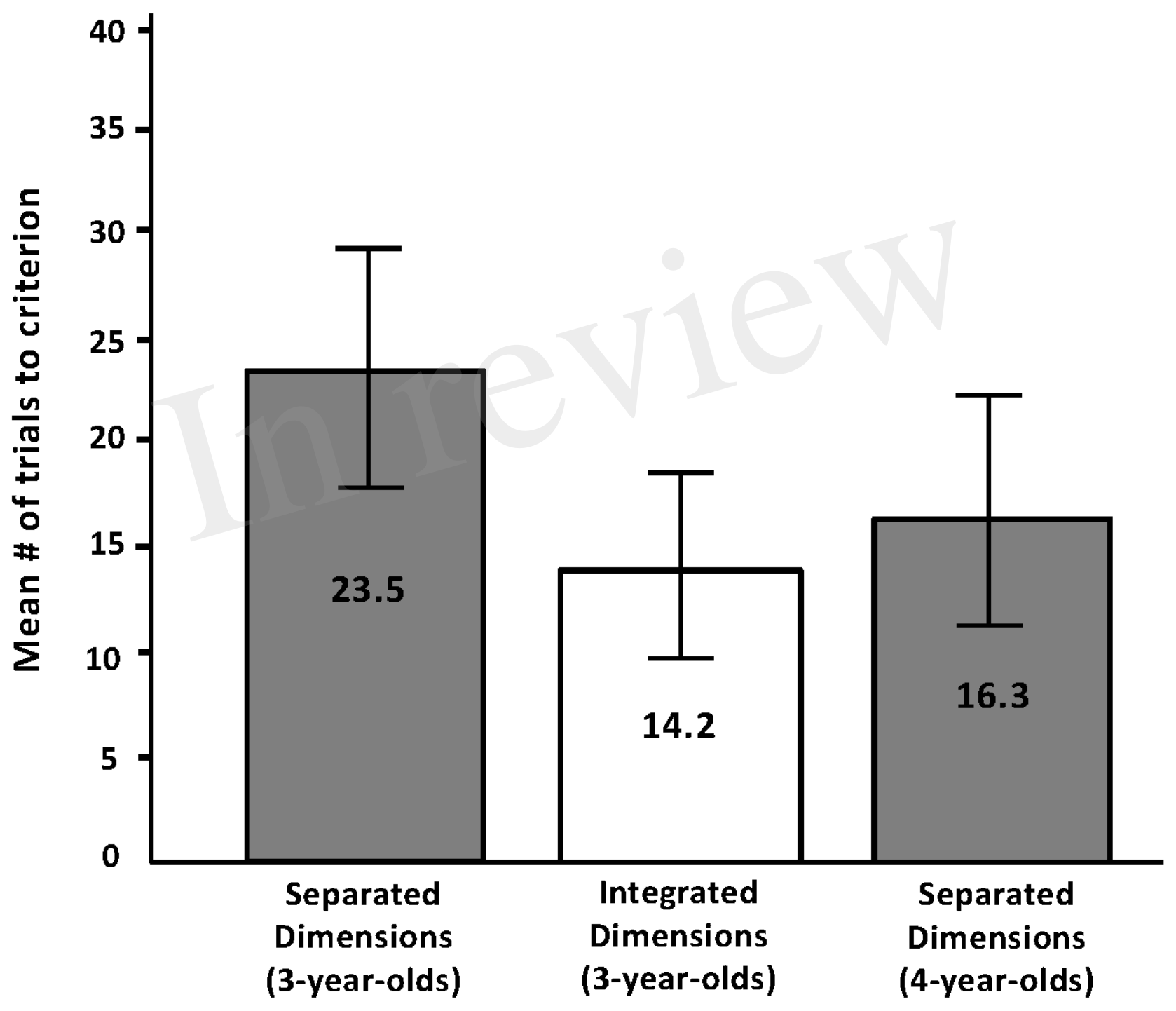

